\title{
Interpreting the high-energy neutrino sky through an angular power spectrum analysis
}

\author{
Ariane Dekker, ${ }^{a, *}$ Shin'ichiro Ando ${ }^{a, b}$ and Marco Chianese ${ }^{c, d}$ \\ ${ }^{a}$ GRAPPA Institute, University of Amsterdam, 1098 XH Amsterdam, The Netherlands \\ ${ }^{b}$ Kavli Institute for the Physics and Mathematics of the Universe (Kavli IPMU, WPI) University of Tokyo, \\ Kashiwa, Chiba 277-8583, Japan \\ cDipartimento di Fisica "Ettore Pancini", Università degli studi di Napoli "Federico II", Complesso Univ. \\ Monte S. Angelo, I-80126 Napoli, Italy \\ ${ }^{d}$ INFN - Sezione di Napoli, Complesso Univ. Monte S. Angelo, I-80126 Napoli, Italy \\ E-mail: a.h.dekker@uva.nl
}

The origin of high-energy neutrinos, observed in the last 10 years by IceCube, is unknown. We gain more insight by studying the expected angular distribution of potential source populations, considering that IceCube observes a neutrino sky consistent with an isotropic distribution. We simulate neutrino skymaps by applying statistical distributions for the fluxes of extra-galactic sources and investigate the sensitivities of current (IceCube) and future (IceCube-Gen2 and KM3NeT) experiments. The angular power spectrum is a powerful probe to assess the angular characteristics of neutrino data and we can constrain rare and bright source classes with current IceCube data. Another potential source is the decay or annihilation of dark matter, suggested by the observed excess in the High-Energy-Starting-Event (HESE) data set of IceCube. These neutrinos are expected to correlate with the galactic centre, allowing us to distinguish between dark matter and astrophysical origin. We apply the angular power spectrum analysis to the HESE data, and set model-independent limits on dark matter properties. This method relies only on the angular distribution of neutrino events and is therefore stable against astrophysical uncertainties. Moreover we perform a sensitivity forecast for IceCube-Gen 2 and KM3NeT exposure for different decaying and annihilating channels. KM3NeT is especially sensitive to low dark matter masses due to its visibility towards the galactic centre.

$37^{\text {th }}$ International Cosmic Ray Conference (ICRC 2021)

July 12th-23rd, 2021

Online - Berlin, Germany

\footnotetext{
${ }^{*}$ Presenter
} 


\section{Introduction}

High-energy neutrinos from astrophysical sources have been detected by IceCube in the last 10 years. The arrival directions of these observed neutrinos are consistent with an isotropic distribution, which suggests a dominant contribution of extragalactic sources. High-energy neutrinos are expected to be produced within or in the surroundings of cosmic-ray accelerators, however the origin of the individual neutrino events remain unknown. Point-like searches have only led to upper limits on contributing source classes and on the flux per individual source. In this work, we study the angular clustering of neutrino events with current and future up-going muon neutrino data, observed with IceCube, IceCube-Gen2 and KM3NeT, by analysing the angular power spectrum (APS). Our goal is to constrain source populations by performing Monte Carlo (MC) simulations, where we apply statistical distributions to assign a flux to each extragalactic neutrino source, instead of using an equal flux for all sources.

Moreover, we test a two-component scenario following the same APS analysis, with an isotropic astrophysical flux, as well as an additional dark matter (DM) signal from decaying or annihilating DM. A two-component scenario has been motivated by an observed tension between two data samples in IceCube. In particular, with the 10-year data-set of through-going (TG) events, a best-fit is found for the spectral index of $\gamma_{\mathrm{TG}}=2.28$ [1], as expected from an extragalactic astrophysical origin. Differently, the 7.5-year High-Energy Starting Events (HESE) data-set, which observes the full sky, prefers a steeper spectrum with a best-fit index of $\gamma^{\mathrm{HESE}}=2.89$ [2]. We test the hypothesis of an isotropic astrophysical flux with the TG best-fit and an additional DM signal, against an isotropic astrophysical flux only from the HESE best-fit. We set constraints on the level of anisotropy induced by the DM component, which translates to limits on the lifetime and cross-section for different DM models.

\section{Extragalactic astrophysical neutrino sky}

We apply the following statistical distribution for the flux of each source,

$$
\frac{d N_{s}}{d F}=N_{\star} \times \begin{cases}\left(\frac{F}{F_{\star}}\right)^{-2.5}, & F_{\star}<F, \\ \left(\frac{F}{F_{\star}}\right)^{-1.5}, & F_{0}<F<F_{\star},\end{cases}
$$

where we assume a broken power-law with slopes based on cosmological and observational considerations. The total number of neutrino sources is represented by $N_{S}$ and the characteristic flux at the break by $F_{\star}$, which corresponds to the characteristic number of sources $N_{\star}$ (see Ref. [3] for further details). We fix the normalization of the distribution to the observed neutrino intensity, $\Phi_{\nu}$, considering that $F_{\star}$ is related to the observed neutrino intensity through $F_{\star} \propto \frac{4 \pi \Phi_{v}}{N_{\star}}$. The characteristic number of sources $N_{\star}$ thus roughly indicates the number of neutrino sources over which the observed intensity is divided. The actual value depends on the source population, and we leave it as a free parameter. Bright source classes like BL Lacs are expected to have small $N_{\star}$ $\left(N_{\star}=6 \times 10^{2}\right)$ and are therefore expected to show more clustering of neutrino events than abundant and weak source classes with larger $N_{\star}$ as for instance starburst galaxies $\left(N_{\star}=10^{7}\right)$. We use these 
angular features to constrain source populations considering that IceCube observes an isotropic neutrino sky.

We distribute the total number of sources, $N_{s}$, randomly over the sky and assign a random flux following the source flux distribution to each source. We use the exposure for through-going muon neutrinos of IceCube and $\mathrm{KM} 3 \mathrm{NeT}$ to obtain the expected number of neutrino detections from each source. Moreover, we add the atmospheric background flux, which shows a nearly isotropic distribution and obtain the total expected neutrino sky maps. We perform MC realizations for $N_{\star}$-values between $\left[10,10^{6}\right]$ with 10 years of observations with IceCube, IceCube-Gen2 and KM3NeT.

\section{Analysis}

We assess the angular power spectrum (APS) to quantify the level of anisotropy on the neutrino sky using anafast tool from the HEALPix package [4, 5]. The neutrino sky is expanded into spherical harmonics, and the APS is described by averaging the expansion coefficients $a_{\ell m}$ as follows,

$$
C_{\ell}=\frac{1}{2 \ell+1} \sum_{m=-\ell}^{\ell}\left|a_{\ell m}\right|^{2} .
$$

In order to test the model with $N_{\star}$, we obtain the $\chi^{2}$-distribution for each $N_{\star}$ from the set of simulations. The probability of obtaining the same or more extreme value of $\chi^{2}$ for each $N_{\star}$ is described by the $p$-value, and we exclude the model at $95 \%$ confidence level (CL), corresponding to $p=0.05$.

\section{Results}

Figure 1 shows the $p$-value results with 10-year IceCube observations [6] (left) and 10-year of IceCube-Gen2 (middle) and KM3NeT (right) exposure. The shaded areas represent the $68 \%$ and $95 \%$ of the MC realizations where we assume an isotropic sky, and the horizontal line shows the exclusion limit with $p=0.05$. With 10 years of IceCube observations, we can constrain $N_{\star}>600$ at $95 \% \mathrm{CL}$, which is also the characteristic number of sources related to BL Lacs, for which a $100 \%$ contribution is thus not allowed by angular information only. With future neutrino data using 10-year of IceCube-Gen2 and KM3NeT exposure, we can set even more significant limits on bright sources. In particular, we find an exclusion of bright sources below $N_{\star}=10^{4}-2 \times 10^{5}$ in the case of IceCube-Gen2 exposure and $N_{\star}=10^{4}-3 \times 10^{4}$ in the case of KM3NeT exposure. Moreover, we can set stringent constraints on the contribution of bright sources such as BL Lacs and FSRQs in future if not observed individually. If, however, we do observe clustering of events in future, we can constrain the contribution of less bright sources like starburst galaxies, and the exclusion tendencies will change as can be found in [7], where we also discuss the detectability of different source classes. We find comparable exclusion trends for IceCube-Gen2 and KM3NeT, which is probably due to the effective area of IceCube-Gen2 being 10 times larger while the angular resolution is improved by a factor of 10 of KM3NeT. 

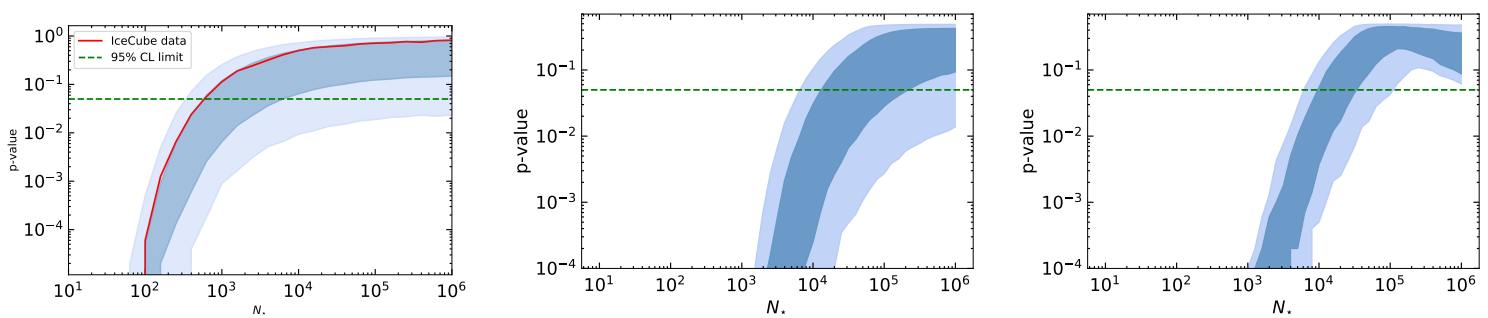

Figure 1: $p$-value results with 10 years of IceCube observations (left), IceCube-Gen2 exposure (middle) and 10 years of KM3NeT exposure (right). The shaded areas represent the $68 \%$ and $95 \%$ bands of the MC simulations, and the horizontal line shows the exclusion limit.

\section{Dark Matter neutrino sky}

We test a two-component scenario of the HESE data set against an extragalactic astrophysical component only, motivated by the observed tension between the HESE and TG events, and we test the second component coming from decaying or annihilating DM. Neutrino events coming from DM will show a correlation with the extended Galactic DM halo, and we can therefore distinguish the two scenarios using the different expected angular distribution of the events.

Following the angular power spectrum analysis, we test a large range of lifetimes and crosssections, of which we present the results in Figure 2 for future sensitivity of neutrino telescopes to decaying (left) and annihilating (right) DM models. We adopt a channel into tau leptons $\left(\tau^{+} \tau^{-}\right)$, a NFW density profile, and cosmological boost factor taken from Ref. [8], however, we test different DM characteristics in Ref. [9] as well. The bands represent the median and upper 95\% values of the $\mathrm{MC}$ runs, constrained at $p$-value of 0.05 . Moreover, the current constraints with 6-year of IceCube data is shown by the solid black lines. We compare our results with bounds from gammaray searches from the Galactic halo HAWC [10] (grey solid lines), global gamma-rays constraints from Ref. [11] (grey dashed lines), and the unitarity constraints on cross-section [12] (black dashed lines). We find that a parameter space not yet constrained by gamma-ray searches is probed, while only the decaying DM case is allowed with respect to the unitarity limit. KM3NeT and IceCubeGen2 show similar result, where KM3NeT is more sensitive to DM models at the lower DM mass range due to its visibility towards the galactic center, while IceCube-Gen2 is more sensitve above $\mathrm{PeV}$. We extend the analysis to masses at lower energies up to $100 \mathrm{GeV}$ for annihilating DM, and find that even at lower energies, the APS analysis offers a robust way to interpret the neutrino sky [13]. Both instruments will be sensitive to the present 7.5-year HESE best-fit of the decaying DM component [14], shown with a black stars in the figure. As the APS analysis relies on angular information only, it is robust against any degeneracies in the neutrino energy spectrum expected from astrophysical sources as well as in DM particles.

\section{Conclusion}

The angular power spectrum analysis offers a powerful probe to interpret the high-energy neutrino sky. In particular, we can assess the contribution of astrophysical source populations in a statistical way, and predict what source classes will be observable with future neutrino data 

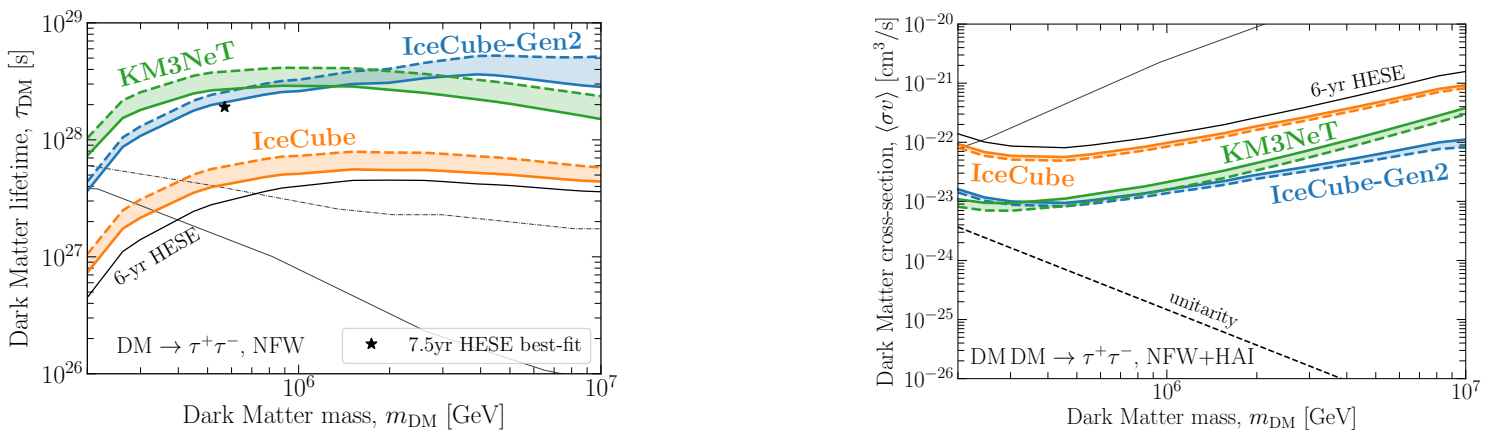

Figure 2: Future sensitivity with 10-year of exposure to decaying (left) and annihilating (right) dark matter models with IceCube, KM3NeT and IceCube-Gen2 experiments. The bands represent the median (dashed lines) and 95\% (solid lines) sensitivity from MC simulations. The current constraints from 6-year HESE data are shown with solid black lines, and the gamma-ray constraints are represented by light grey lines: solid with HAWC galactic halo searches [10] and dot-dashed (left panel) from Ref. [11].

using angular information only. We set constraints on the characteristic number of sources $N_{\star}$ for IceCube, IceCube-Gen2 and KM3NeT by using that IceCube observes a distribution of neutrino events which is consistent with isotropy. With current 10 year of TG IceCube data, we constrain bright source populations with $N_{\star}>600$ at $95 \% \mathrm{CL}$, which interestingly is also the characteristic number of sources for BL Lacs. With future neutrino data, we expect to set a significant constraints on source populations such as BL Lacs and FSRQs in the case of an isotropic distribution. However, if we observe clustering of events in the future, weak source classes could be constrained too.

Moreover, we test a two-component interpretation of the HESE events, with a second component coming from decaying or annihilating DM. We set limits on the DM lifetime and cross-section with current 6-year IceCube HESE data, as well as provide future sensitivities with 10-year of IceCube, IceCube-Gen2 and KM3NeT exposure. With the APS analysis, a DM signal can be distinguished from potentially hidden astrophysical sources, and moreover, test the present best-fit of a decaying DM component.

\section{References}

[1] ICECube collaboration, Measurement of the Diffuse Astrophysical Muon-Neutrino Spectrum with Ten Years of IceCube Data, in ICRC2019, 2019 [1908.09551].

[2] ICECube collaboration, Characterization of the Astrophysical Diffuse Neutrino Flux with IceCube High-Energy Starting Events, in ICRC 2019, 2019 [1907 . 11266].

[3] S. Ando, M.R. Feyereisen and M. Fornasa, How bright can the brightest neutrino source be?, Phys. Rev. D95 (2017) 103003 [1701.02165].

[4] A. Zonca, L. Singer, D. Lenz, M. Reinecke, C. Rosset, E. Hivon et al., healpy: equal area pixelization and spherical harmonics transforms for data on the sphere in python, Journal of Open Source Software 4 (2019) 1298. 
[5] K. Gorski, E. Hivon, A. Banday, B. Wandelt, F. Hansen, M. Reinecke et al., HEALPix - A Framework for high resolution discretization, and fast analysis of data distributed on the sphere, Astrophys. J. 622 (2005) 759 [astro-ph/0409513].

[6] M. Aartsen, M. Ackermann, J. Adams, J. Aguilar, M. Ahlers, M. Ahrens et al., Time-integrated neutrino source searches with 10 years of icecube data, Physical Review Letters 124 (2020).

[7] A. Dekker and S. Ando, Angular power spectrum analysis on current and future high-energy neutrino data, JCAP 1902 (2019) 002 [1811.02576].

[8] N. Hiroshima, R. Kitano, K. Kohri and K. Murase, High-energy neutrinos from multibody decaying dark matter, Phys. Rev. D97 (2018) 023006 [1705 . 04419].

[9] A. Dekker, M. Chianese and S. Ando, Probing dark matter signals in neutrino telescopes through angular power spectrum, 1910.12917.

[10] HAWC collaboration, A Search for Dark Matter in the Galactic Halo with HAWC, JCAP 1802 (2018) 049 [1710. 10288].

[11] T. Cohen, K. Murase, N.L. Rodd, B.R. Safdi and Y. Soreq, -ray Constraints on Decaying Dark Matter and Implications for IceCube, Phys. Rev. Lett. 119 (2017) 021102 [1612.05638].

[12] K. Griest and M. Kamionkowski, Unitarity Limits on the Mass and Radius of Dark Matter Particles, Phys. Rev. Lett. 64 (1990) 615.

[13] S. Basegmez du Pree, C. Arina, A. Cheek, A. Dekker, M. Chianese and S. Ando, Robust limits from upcoming neutrino telescopes and implications on minimal dark matter models, Journal of Cosmology and Astroparticle Physics 2021 (2021) 054.

[14] M. Chianese, D.F.G. Fiorillo, G. Miele, S. Morisi and O. Pisanti, Decaying dark matter at IceCube and its signature on High Energy gamma experiments, 1907.11222. 